\title{
EXPLORING THE TURBULENT URBAN BOUNDARY BY USE OF LIDARS AND MICROWAVE RADIOMETERS
}

\author{
Mark Arend $^{1 *}$, Ivan Valerio ${ }^{1}$, Stephen Neufeld ${ }^{1}$, Raymond Bishir ${ }^{2}$, Younghu Wu ${ }^{1}$ \\ Fred Moshary ${ }^{1}$, David Melecio-Vazquez ${ }^{2}$, Jorge Gonzalez ${ }^{2}$ \\ ${ }^{1}$ Optical Remote Sensing Laboratory, Electrical Engineering Department and NOAA CREST \\ CENTER, The City College of New York, New York, NY10031*EMAIL: marend@,ccny.cuny.edu \\ ${ }^{2}$ Mechanical Engineering Department and NOAA CREST CENTER, The City College of New \\ York, New York, NY 10031
}

\begin{abstract}
A Doppler lidar has been developed using fiber optic based technologies and advanced signal processing techniques. Although this system has been operated in a scanning mode in the past, for this application, the system is operated in a vertically pointing mode and delivers a time series of vertical velocity profiles. By cooperating the Doppler lidar with other instruments, including a back scatter lidar, and a microwave radiometer, models of atmospheric stability can be tested, opening up an exciting path for researchers, applied scientists and engineers to discover unique phenomena related to fundamental atmospheric science processes. A consistent set of retrievals between each of these instruments emphasizes the utility for such a network of instruments to better characterize the turbulent atmospheric urban boundary layers which is expected to offer a useful capability for assessing and improving models that are in great need of such ground truth.
\end{abstract}

\section{INTRODUCTION}

Research in turbulent boundary layer dynamics has been and will likely continue to be of interest to scientists and engineers because of the wide applications across many disciplines. However, defining turbulence and deriving closed form physical solutions that are based on strict axiomatic and physical principles continues to be an unsolved challenge even though much advancement in modern science and mathematics has been made [1]. Experimental methods that utilize Doppler lidars are very useful for investigating flows since the motion as well as the constituent concentration can be tracked simultaneously, thus providing additional metrics for assessing the flow dynamics. When applied to real world situations such as the urban atmospheric boundary layer, the possibility exists for there being relevant applications that depend on robust and field-able instruments and networks. A fiber based Doppler lidar system [2-7], a back scatter Raman lidar system [8] and a network of other Ground based remote sensing instruments (including a microwave radiometer for measuring temperature and water vapor profiles) are included in a set of instruments comprising the NYCMetNet [9], developed by members of the Optical Remote Sensing Lab at the City College of New York. This suite of instruments are sometimes (but not always) co-operating and when there is overlap, there is an opportunity to look closely at the consistencies between the instruments and expose any inconsistencies by following a time series of the vertical profiles and comparing these visualizations with one another.

\section{METHODOLOGY}

COHERENT DOPPLER LIDAR: The Coherent Doppler lidar has been constructed and tested at CCNY. The system consists of a 1542 $\mathrm{nm} \mathrm{CW}$ laser fiber laser that is split in to two paths such that one path is used for a local oscillator and the other path is amplified by a single mode erbium doped fiber amplifier EDF and then modulated by an acousto-optic modulator, producing $200 \mathrm{nsec}$ pulses at $20 \mathrm{kHz}$. These pulse are amplified further with a final amplifier and sent to an optical circulator before be launched in to the 4 inch clear aperture 
telescope from the single mode fiber. The back scattered light from the atmosphere is gathered by the telescope, passed through the circulator and mixed with the local oscillator with a $2 \times 2$ coupler. A $12 \mathrm{~h}$ MHZ bandwidth balanced photodetector followed by TIA amplifiers detect the signal which is then sampled by a 400 $\mathrm{MS} / \mathrm{sec} 12$ but ADC. These signals are processed with a FPGA that calculates gated Fourier transforms (or autocorrelations depending on the chosen mode) and accumulate power spectra for further processing on a host computer.

\section{DIRECT DETECTION BACKSCATTER}

LIDAR : The direct detection backscatter lidar was made using a Q-switched Spectra-Physics Quanta Ray Pro230 Nd-YAG with variable output power up to $475 \mathrm{~mJ} @ 532 \mathrm{~nm}, 950 \mathrm{~mJ}$ @ $1064 \mathrm{~nm}$ and $300 \mathrm{~mJ} @ 355 \mathrm{~nm}$. This system currently has five channels with wavelengths at $1064 \mathrm{~nm}, 532 \mathrm{~nm}, 355 \mathrm{~nm}$, including a Raman channel at $407 \mathrm{~nm}$ for water with a second Raman channel at 387 for nitrogen. The repetition rate is $30 \mathrm{~Hz}$ with $1-2$ ns pulse duration @ $532 \mathrm{~nm} ;<0.5 \mathrm{mrad}$ beam divergence. The beam is on axis to the telescope. See ref. WU for more information.

MICROWAVE RADIOMETER: The Radiometrics Profiling Radiometer, model MP$3000 \mathrm{~A}$, is also operated at CCNY in Manhattan and produces vertical profiles from the surface to $10 \mathrm{~km}$, producing high-resolution temperature, relative humidity and water vapor profiles, and low-resolution liquid profiles. The MP-3000A incorporates two radio frequency (RF) subsystems in the same cabinet. These RF subsystems share the same antenna and antenna pointing system. The temperature profiling subsystem utilizes sky brightness temperature observations at selected frequencies between 51 and $59 \mathrm{GHz}$. The water vapor profiling subsystem utilizes sky brightness temperature observations at selected frequencies between 22 and $30 \mathrm{GHz}$. Atmospheric stability is related to the actual sounding relative to a critical sounding which depends on how much water vapor is in the air. Fig. 1 shows an illustration of the dry adiabatic sounding and shows how stability is referenced to the dry adiabatic laps rate $\Gamma_{\mathrm{d}}$.
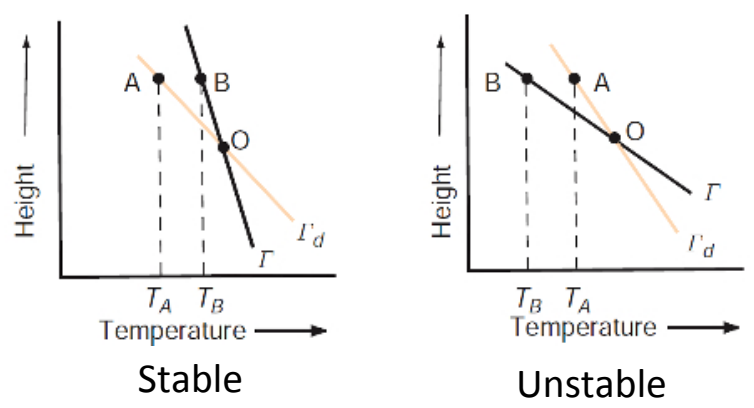

Fig. 1 Stable and unstable sounding relative to the dry adiabatic laps rate $\Gamma_{\mathrm{d}}$.

The virtual potential temperature is a convenient measurement for the air since it takes into account the variability in density between dry air and moist air ("virtual"), and difference in temperatures due to pressure variation.

The Radiometer is used to derive the virtual potential temperature from the analytical profiles of temperature, $\mathrm{T}$, and relative humidity, $\mathrm{RH}$. From the observed temperature profiles, the potential temperature and the saturation pressure are calculated. From the observed relative humidity profiles, the saturation mixing ratio and the actual mixing ratio values are generated. These values are then used to derive virtual potential temperature generates a vertical profile that can be used for classifying the stability of the atmosphere. If the virtual potential temperature increases with elevation the atmosphere is considered stable. An unstable (or convective) atmosphere is defined for conditions in which the virtual potential temperature decreases with height. Neutral stability is defined when there is no change in virtual potential temperature with height.

RESULTS: Figs. 2-7 shows the processed signals from each of these three instruments for a particular day (10/31/2014). Colored boxes represent the time and height where the data from each instrument is sampled since both the lidars are not continuously operated. Magenta color represents the coherent Doppler Lidar sampling and the Green color represents the direct detection lidar sampling. 
Level-1 Product: Range-Corrected Elastic-Scattering Return at 532nm 20141031 CCNY-lidar total attenuated backscatter $\left(\mathrm{km}^{-1} \mathrm{sr}^{-1}\right)$ at 532-nm $90^{-3}$

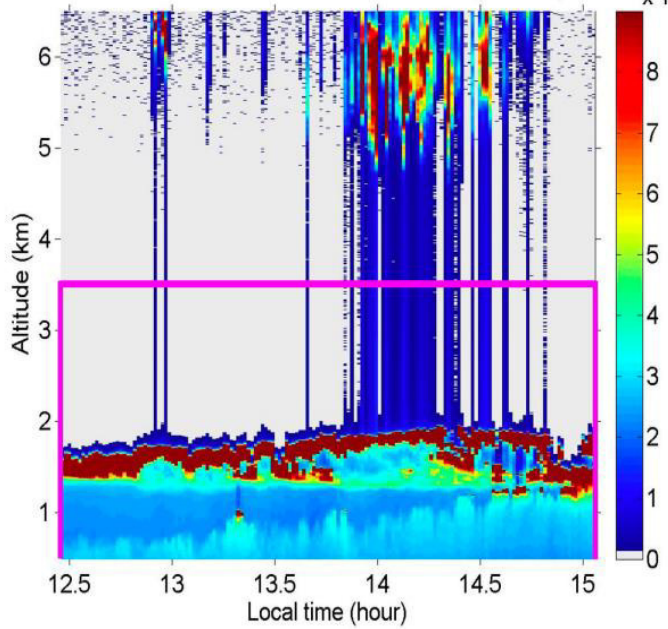

Fig. 2 Direct detection lidar at $532 \mathrm{~nm}$

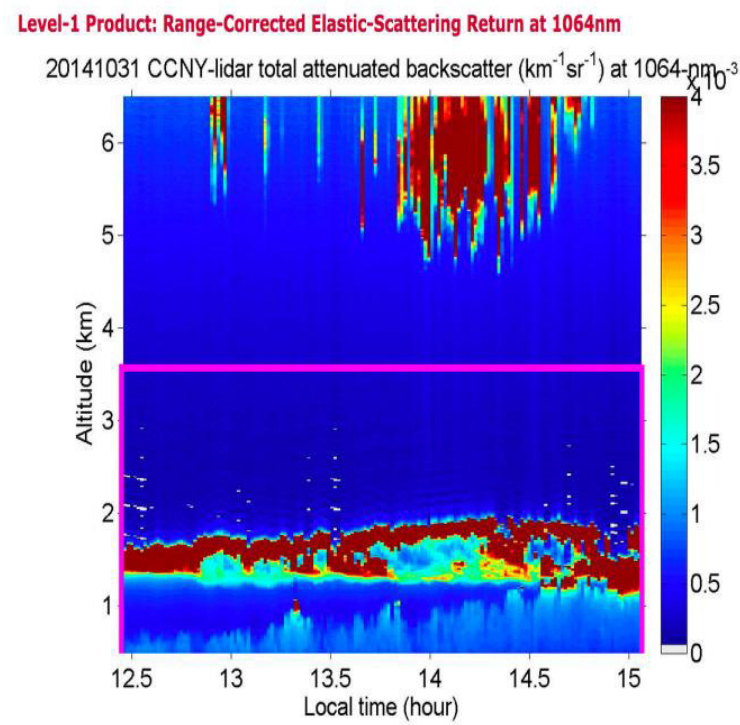

Fig. 4 Direct detection Lidar at $1064 \mathrm{~nm}$

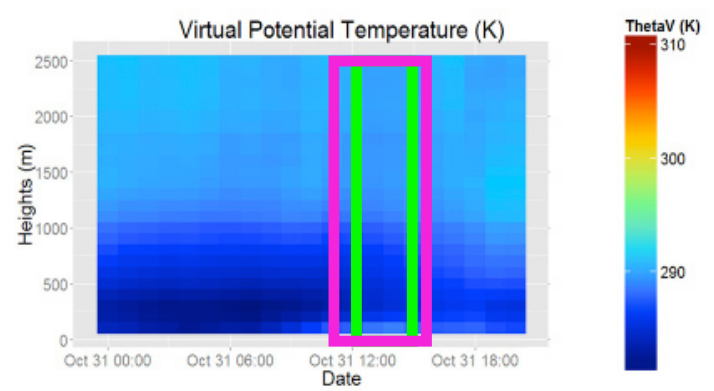

Fig. 6 Virtual Potential Temperature

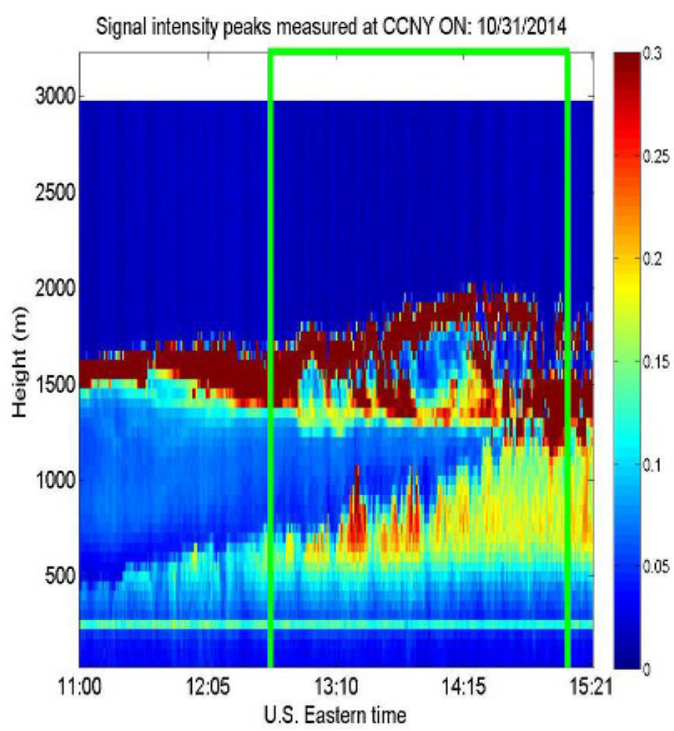

Fig. 3 Coherent Detection Doppler lidar Signal Intensity

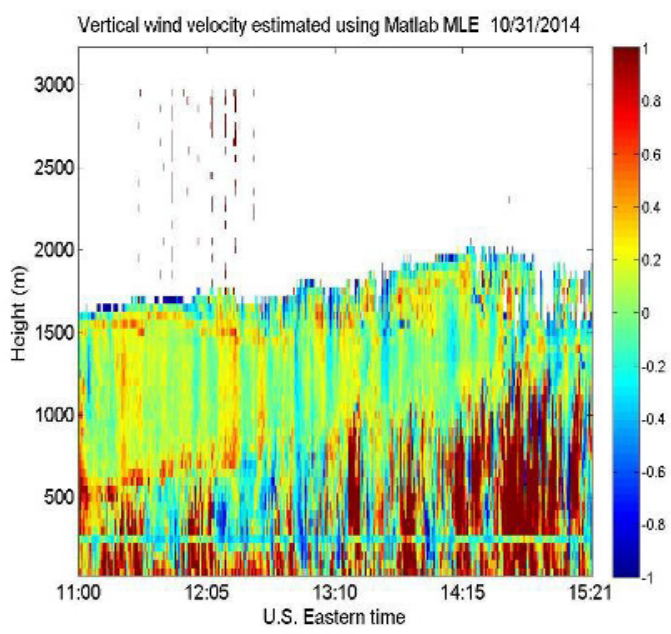

Fig. 5 Coherent Detection Lidar Vertical Velocity $(\mathrm{m} / \mathrm{s})$

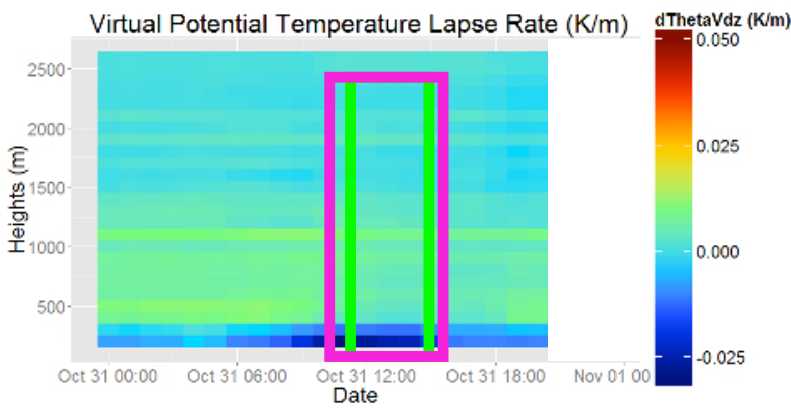

Fig. 7 Virtual Potential Temperature Laps rate 
DISCUSSION: On this particular day (Oct 31, 2014) the stability of the atmosphere was not being forced by any significant mean surface winds and the upper air winds were sufficiently quite so as to not cause significant transport of aerosols horizontally as observed by other instruments in the CCNY NYCMetNet. This then simplifies the interpretation of the temporal evolution of the three instruments as follows: The large thermal gradient of the virtual potential temperature (Fig. 6) a few hours before and after noon shows strong instability which would be expected to cause a significant uplifting. This gradient is shown even more dramatically in the laps rate (Fig. 7, the negative of the derivative of virtual potential temperature vs. height) plot. This episode is consistent with the strong vertical motion that begins to evolve during the same time period (Fig 5) which dramatically increases during the latter part of this time period. The growth of the boundary layer and the increased presence of aerosols throughout this time period is very evident in Figs. 2, 3 and 4. Figs. 2, 3 and 4 are also consistent with each other in depicting the presence of the aerosol plume that lifts up under the clouds at 1.5 to $2 \mathrm{~km}$.

CONCLUSION: A coherent Doppler lidar, a direct detection back scatter lidar and a microwave radiometer have been operated cooperatively to demonstrate that each of these instruments respond to a dynamically changing atmospheric boundary layer stability condition in a manner that is consistent across the suite of instruments. This inspires further studies to demonstrate these principles under other dynamically changing conditions so that a stability climatology can be documented which should have an impact on model development.

ACKNOWLEDGEMENTS: Supported in part by NOAA Cooperative Agreement No: NA11SEC4810004

\section{REFERENCES}

[1] Gorban A.N.; Karlin, I. "Hilbert's 6th Problem: Exact and Approximate Hydrodynamic Manifolds for Kinetic
Equations” Bull. Amer. Math. Soc. 51 (2014), 187-246

[2] M. Arend, S. Abdelazim, M. Lopez and F. Moshary, "A comparison of two embedded programming techniques for high rep rate coherent Doppler lidars," in SPIE, 2013.

[3] S. Abdelazim, D. Santoro, M. Arend, F. Moshary and S. Ahmed, "Field programmable gate array processing of eye-safe all-fiber coherent wind Doppler lidar return signals," in SPIE, Remote Sensing, Prague, 2011.

[4] S. Abdelazim, D. Santoro, M. Arend, F. Moshary and S. Ahmed, "Wind Velocity Estimate and Signal to Noise Ratio Analysis of an All Fiber Coherent Doppler Lidar System," in 16th Coherent Laser Radar Conference, Long Beach, California, June, 2011.

[5] S. Abdelazim, D. Santoro, M. Arend, F. Moshary and S. Ahmed, "All-fiber Coherent Doppler LIDAR for Wind Sensing," in 5th Symposium on Lidar Atmospheric Applications, 91st American Meteorological Society Annual Meeting, Seattle, Washington, 2011.

[6] S. Abdelazim, D. Santoro, M. Arend, F. Moshary and S. Ahmed, "Development of Allfiber Coherent Doppler LIDAR System for Wind Sensing," in 15th Symposium on Meteorological Observation and Instrumentation, 90th AMS Annual Meeting, Atlanta, GA, 2010.

[7] S. Abdelazim, D. Santoro, M. Arend, F. Moshary and S. Ahmed, "All-fiber Coherent Doppler LIDAR for Wind Sensing," in 15th Annual Coherent Laser Radar Conference, Toulouse, France, 2009.

[8] Wu, Y., Chaw, S. Gross, B., Moshary, F., Ahmed, S. "Low and optically thin cloud measurements using a Raman- Mie lidar" Applied Opt. 48, 1218-1227, 2009.

[9] Committee on Urban Meteorology: Scoping the Problem, Defining the Needs; Board on Atmospheric Sciences and Climate; Division on Earth and Life Sciences, National Research Council, 2012, Urban Meteorology: Forecasting, Monitoring, and Meeting Users' Needs, Washington, DC: The National Academies Press, p54, http://nycmetnet.ccny.cuny.edu 